\title{
In the thick of it: peroral endoscopic myotomy with intramuscular tunneling in end-stage achalasia
}

\author{
Georgios Mavrogenis ${ }^{a}$, Louis M. Wong Kee Song ${ }^{b}$, Fateh Bazerbachic \\ Mediterraneo Hospital, Athens, Greece; Mayo Clinic, Rochester, Minnesota, USA; CentraCare Digestive Center, St. \\ Cloud Hospital, Minnesota, USA
}

An 80-year-old man with end-stage achalasia presented with aspiration pneumonia, weight loss, and debilitation. He was not deemed a surgical candidate for palliative esophagectomy. Previous treatment with dilation was unsucessful. A posterior-like anterior peroral endoscopic myotomy (POEM) was performed [1,2]. Following mucosotomy and an intial 2-cm submucosal tunnel creation, excessive fibrosis within the submucosa was encountered. The fibrosis-laden submucosa elevated the risk of inadvertent mucosal injury and impeded subsequent dissection. We therefore decided to veer the axis of dissection below the dissection plane and created an intramuscular tunnel through the hyperplastic muscularis propria (Fig. 1,2). After $4 \mathrm{~cm}$ of intramuscular tunneling, the submucosal space became accessible again, and the axis of dissection was redirected between the mucosa and the muscularis propria. Full-thickness myotomy of the cardia was performed and the mucosotomy site was closed. The patient was discharged after $48 \mathrm{~h}$. He had an uneventful recovery and remains asymptomatic at 6 months of follow up.

Mucosal injury is a recognized complication of POEM, occurring in up to $5 \%$ of cases [3]. Mucosal and submucosal fibrosis is a major risk factor for such a complication and typically occurs following prior therapies, such as botulinum toxin injection and dilation, or in the setting of long-standing achalasia with hyperkeratotic mucosa. In this report, we present a rescue approach when encountering severe mucosal/submucosal fibrosis. Instead of aggressive dissection through the plane of

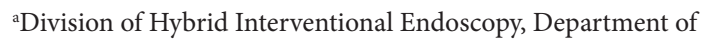
Gastroenterology, Mediterraneo Hospital, Athens, Greece (Georgios Mavrogenis); ' Division of Gastroenterology and Hepatology, Mayo Clinic, Rochester, Minnesota, USA (Louis M. Wong Kee Song); 'Interventional Endoscopy Program, CentraCare Digestive Center, St. Cloud Hospital, St. Cloud, Minnesota, USA (Fateh Bazerbachi)

Conflict of Interest: None

Correspondence to: Georgios Mavrogenis, Director of Hybrid Interventional Endoscopy, Department of Gastroenterology, Mediterraneo Hospital, Ilias 12, 16675, Glyfada, Athens, Greece, e-mail: mavrogenis@gmail.com

Received 3 April 2021; accepted 15 April 2021; published online 2 July 2021

DOI: https://doi.org/10.20524/aog.2021.0657

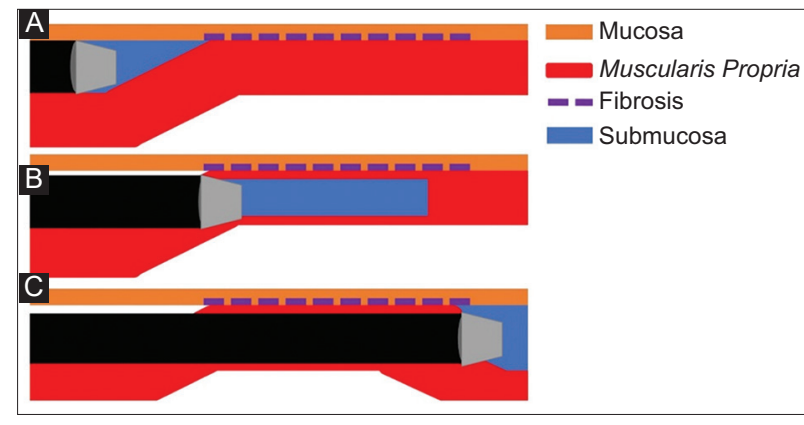

Figure 1 Schematic representation of intramuscular tunnelling. (A) Initiation of the submucosal tunnel. (B) Deviation of the axis of dissection through the muscularis propria. (C) At the end of the fibrotic segment, the submucosal space is reaccessed

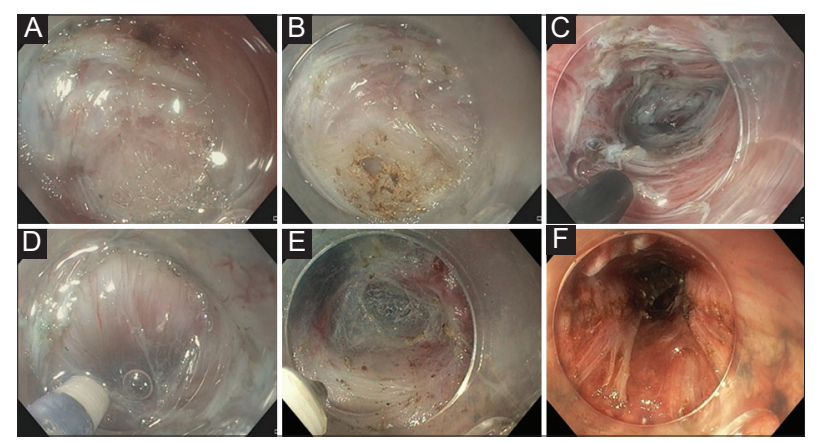

Figure 2 Endoscopic images of intramuscular tunnelling. (A) Excessive fibrosis. (B) Creation of a small hole through the muscularis propria.

(C) Intramuscular tunnel. (D) Reaccessing the submucosal space.

(E) Submucosal tunnel at the cardia. (F) Full-thickness myotomy

fibrosis, which can result in mucosal injury, we proceeded with intramural dissection of the thickened muscularis propria.

\section{References}

1. Mavrogenis G, Bazerbachi F, Tsevgas I, Zachariadis D. "Posterior-like" anterior per-oral endoscopic myotomy. VideoGIE 2019;4:194-196.

2. Mavrogenis G, Antoniou P, Tsevgas I, Zachariadis D. "Posteriorlike" anterior peroral endoscopic myotomy: a novel concept. Ann Gastroenterol 2018;31:635.

3. Nabi Z, Reddy DN, Ramchandani M. Adverse events during and after per-oral endoscopic myotomy: prevention, diagnosis, and management. Gastrointest Endosc 2018;87:4-17. 